\title{
INVESTIGACIONES
}

\section{El rol de las creencias en los modelos de aprendizaje auto-regulado y su relación con el aprendizaje del dibujo*}

\author{
The role of beliefs in self-regulated learning models \\ and its relation with drawing learning \\ O papel das crenças nos modelos de aprendizagem autorregulados \\ e sua relação com a aprendizagem do desenho
}

\author{
Marianela Noriega Biggio ${ }^{1}$,Stella Maris García ${ }^{2}$,Stella Maris Vázquez ${ }^{3}$ \\ ${ }^{1}$ Centro de Investigaciones en Antropología Filosófica y Cultural (CIAFIC), Facultad de Arquitectura, Diseño y \\ Urbanismo de la Universidad de Buenos Aires (FADU- UBA), +54 11 47775280, marianelanoriega@ gmail.com \\ ${ }^{2}$ Facultad de Arquitectura, Diseño y Urbanismo de la Universidad de Buenos Aires (FADU- UBA), \\ +54 114925 2277, stellagarcia@netizen.com.ar \\ ${ }^{3}$ Centro de Investigaciones en Antropología Filosófica y Cultural (CIAFIC), Consejo Nacional de \\ Investigaciones Científicas y Técnicas (CONICET), +54 114777 5280, stellavazquez@gmail.com
}

\begin{abstract}
RESUMEN
Se evaluó la objetividad del juicio que los alumnos hacen acerca de su habilidad para el dibujo a mano alzada, al comienzo de sus estudios y la relación entre la creencia y los componentes del aprendizaje auto-regulado.

La muestra de 300 sujetos está compuesta por alumnos que cursan el Ciclo Básico Común en la Facultad de Arquitectura Diseño y Urbanismo de la Universidad de Buenos Aires. La objetividad de la creencia en la propia habilidad se ha verificado parcialmente, para el caso del grupo de sujetos que se manifiestan negativamente al respecto. En el grupo que se evaluó erróneamente como bueno o muy bueno, este error parece ligarse con un estilo de aprendizaje superficial, lo que hace pensar que estos sujetos tienen menos conciencia de sus límites. A partir de los resultados se discute la relación entre agentividad y creencia de autoeficacia específica y se señalan líneas de intervención pedagógica.
\end{abstract}

Palabras clave: creencia, aprendizaje auto-regulado, dibujo, estilos de aprendizaje.

\section{ABSTRACT}

An empirical study is reported, whose goal is twofold. In the first place, it seeks to assess the objectivity of the judgements students make about their hand-drawing skills at the start of their university education. It then focuses on the particular relation between beliefs and self-regulated learning. The sample is made up of 300 students enrolled on the basic course taught at the Architecture, Design and Urban Planning School of the University of Buenos Aires. The subjects' negative beliefs about their own ability have been objectively verified, whereas those who rated themselves as 'good' or 'very good' have been proved wrong. Their mistake seems to be linked to a superficial learning style, which would suggest that students showing less inclination for reflection as well as low processing and self-regulation strategies levels are less aware of their limitations. The relation between agency and specific self-efficacy is discussed. Instructional guidelines are put forward.

Keywords: beliefs, self-regulated learning, drawing, learning styles.

En el marco de UBACyT 2012-2014: Competencia espacial para el proyecto del hábitat. Experiencia didáctica en el aprendizaje del dibujo. Equipo de Investigación Directora: Arq. Stella Maris García. Investigadores: Arq. Carlos Barone; Arq. Mariana Basiglio; Arq. Marianela Noriega Biggio; Arq. Laura Oliva; Dra. en Filosofía Stella Maris Vázquez. 


\section{RESUMO}

Avaliou-se a objetividade do julgamento que os estudantes fazem a respeito de sua habilidade para o desenho à mão livre no começo de seus estudos e a relação entre a crença e os componentes da aprendizagem autorregulada. A mostra de 300 sujeitos está composta por estudantes que estudam no Ciclo Básico Comum da Faculdade de Arquitetura, Desenho e Urbanismo da Universidade de Buenos Aires. A objetividade da crença na própria habilidade foi verificada, parcialmente, para o caso do grupo de sujeitos que se manifestam negativamente a respeito do assunto. No grupo que se avaliou erroneamente como bom ou muito bom, o erro parece unir-se com um estilo de aprendizagem superficial, o que faz pensar que estes sujeitos têm menos consciência de seus limites. A partir dos resultados discute-se a relação entre agentividade e crença de autoeficácia específica e assinalam-se linhas de intervenção pedagógica.

Palavras-chave: Crença. Aprendizagem autorregulada. Desenho. Estilos de aprendizagem.

\section{INTRODUCCIÓN}

El aprendizaje autorregulado (AAR) es un concepto-sistema que refiere al manejo general de la propia conducta académica, a través de procesos interactivos entre distintos sistemas de control: atención, meta-cognición, motivación, emociones, acción y control volitivo (Boekaerts y Niemivirta, 2000; Boekaerts y Corno, 2005). Paul Pintrich lo define como un proceso activo-constructivo por el que el alumno fija sus propias metas de aprendizaje, monitorea, regula y controla su adquisición de conocimientos, motivación y conductas, guiado por las metas y los rasgos contextuales del entorno (Pintrich, 2000; Pintrich y Zusho, 2002; Schunk, 2005). Esos procesos, implicados en la autorregulación académica, están atravesados por las creencias del sujeto acerca de lo que es aprender y de su propia habilidad para ello.

Zimmerman (2000) explicita que en la perspectiva socio-cognitiva, el AAR se considera un proceso interactivo que entraña no sólo habilidades de conducta para manejar las contingencias ambientales, sino también el conocimiento y un sentido de auto-eficacia (personal agency) para activar esas habilidades en contextos relevantes. La autorregulación refiere a pensamientos, sentimientos y acciones auto-generadas que se planifican y adaptan para el logro de metas personales.

Según Bandura, la autoeficacia se define como la creencia en la propia capacidad para organizar y ejecutar los cursos de acción requeridos para producir determinados resultados (Bandura, 1997). Por otra parte, el autor sostiene que la creencia de autoeficacia puede referirse a dominios específicos, es decir que las personas pueden juzgar de modo diferencial su habilidad para desempeñarse en distintos ámbitos. A su vez, los distintos campos de aprendizaje requieren de habilidades tanto generales como específicas, y parecería evidente que las creencias relativas a la posesión de estas últimas deben tener directa relación con los niveles de desempeño.

En estudios experimentales (Salanova y Llorens, 2010), se ha comprobado que la creencia en la autoeficacia influye sobre el nivel de compromiso de modo indirecto, porque impacta en el afecto de modo positivo, suscita entusiasmo y permite así que la acción se sostenga en el tiempo. Al respecto, cabría preguntarse si frente a una creencia errónea en la autoeficacia, la relación no es inversa, es decir, si el nivel de compromiso y la acción sostenida en el tiempo no se ven afectados por esta creencia errónea.

Mancini (Mancini et al., 2013) señala que las convicciones relativas a la autoeficacia son indicadores de agentividad, porque influyen en la resistencia a los obstáculos y al nivel 
de estrés que se experimenta al afrontar requerimientos de tareas y esa influencia se ejerce a través de la regulación de procesos cognitivos, motivacionales y afectivos.

Nuestro interés se ha centrado en un tipo de autoeficacia específica, referida a una habilidad que es básica para el aprendizaje de los contenidos referidos a las carreras de Diseño: la habilidad para el dibujo a mano alzada.

En la actualidad se verifica una revalorización de la importancia de este tipo de dibujo, en particular en las carreras de Diseño, que se sustenta tanto en hallazgos relacionados con la Psicología Cognitiva como en posiciones teóricas referidas a tesis antropológicas y epistemológicas. En efecto, desde la Psicología Cognitiva se subraya la relación entre comprender y

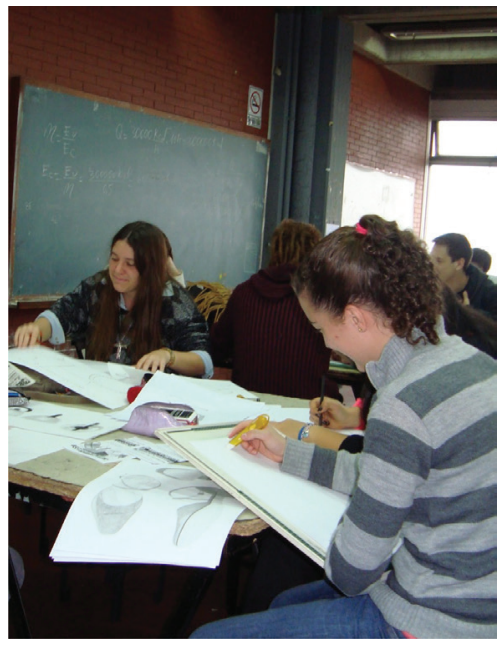
hacer, en tanto que desde la Antropología se acude a la íntima relación entre corporeidad y mente en el hombre, que permite hablar de un conocimiento que es siempre encarnado, que no sólo se inicia en la sensibilidad, sino que tiene siempre la última piedra de toque en las funciones sensibles. En el caso del diseño, estas tesis hallan particular sustento en la Filosofía Fenomenológica, a partir del cuestionamiento de la tesis cartesiana según la cual el hombre es la razón, el pensamiento discursivo, independizado de las funciones sensibles, de todo lo que refiere al cuerpo. Estas tesis han atravesado más de cuatro siglos, impregnando las concepciones y el quehacer cultural con los presupuestos del Racionalismo.

En relación con el quehacer del arquitecto y, de modo más amplio, con el de todo el que diseña, son muchas las voces que se alzan, revalorizando el rol esencial de la corporeidad. Así, Pallasmaa, un referente cuyos trabajos tienen particular relevancia en la temática que nos ocupa, ha señalado que la cuestión teórica acerca de la concepción del conocimiento sensible, el predominio que se ha dado a la visión, por encima de otras fuentes de conocimiento sensible, en particular el tacto, ha influido de modo notable en la forma de pensar y enseñar en el ámbito del diseño y de la arquitectura (Pallasmaa, 2005), en la medida en que la obra del arquitecto o diseñador, ligada esencialmente al espacio, se experimenta en su esencia material y espiritual de modo integrado. Al respecto, el autor cuestiona el uso generalizado de la tecnología, señalando que:

Las imágenes por ordenador tienden a aplanar nuestras magníficas, multisensoriales, simultáneas y sincrónicas capacidades de imaginación al convertir el proceso del proyecto en una manipulación visual pasiva, un viaje de la retina. El ordenador crea una distancia entre el autor y el objeto, mientras que el dibujo a mano, así como la confección de maquetas, colocan al proyectista en un contacto háptico con el objeto o el espacio. En nuestra imaginación, el objeto se sujeta con la mano y se mantiene simultáneamente dentro de la cabeza, y nuestros cuerpos modelan la imagen figurada y proyectada físicamente (Pallasmaa, 2009: 86).

Algo análogo podría decirse respecto de la fotografía, recurso usado por muchos alumnos para sustituir la toma de apuntes en clase: en vez de hacer simultáneamente con el profesor lo que éste dibuja en el pizarrón, toman fotografías del mismo al final de la explicación. 
La importancia del dibujo manual tiene su fundamento más profundo en el modo de ser y de conocer que son propios del hombre. Dice Trachana que:

Aprender a dibujar para proyectar es adquirir hábitos operativos -comprensivos y ejecutoresnuevos, no sólo aprender a dibujar como mera disciplina propedéutica [...] En la configuración arquitectónica, la implicación del cuerpo, brazo, manos, dedos, el cuerpo como soporte y como modelo es esencial. Dibujar-modelar-proyectar la virtualidad envolvente de la arquitectura como espacio vivencial implica acciones-reacciones de alto contenido subjetivo-sensitivo-emocional [...] Por eso, el aprendizaje inicial es experimentar con gestos, acciones, medios... que permitan liberar las manos, sentir el placer de su fluidez movimental y sorprenderse de su capacidad formante $[\ldots]$ La manipulación de diversos materiales y herramientas para dibujar y construir modelos intensifican la percepción táctil y permiten completar el conocimiento a través de la visión [...] Necesitamos las manos para ver, para completar el resto de los sentidos, para llenar de la autenticidad de la materia y de la construcción el hecho arquitectónico (Trachana, 2012: 292).

La habilidad para el dibujo a mano alzada contribuye a desarrollar el dominio de la espacialidad, que es un requisito fundamental para los alumnos que se inscriben en carreras de Arquitectura y Diseño, de allí que su adecuada ejercitación debe ser un objetivo esencial en estas carreras, lo que motivó nuestro interés por explorar dicha habilidad, así como la conciencia de la misma, en un grupo de alumnos de primer año.

Las preguntas centrales que nos hacemos son:

¿Cuál es el juicio que los alumnos hacen acerca de su habilidad para el dibujo a mano alzada, en el momento de iniciar sus estudios en este ámbito?

¿Qué nivel de objetividad tiene dicha creencia?

¿Qué relación hay entre la creencia en la propia habilidad para el dibujo a mano alzada y los componentes del aprendizaje autorregulado, cuya combinación constituye el estilo de aprendizaje de

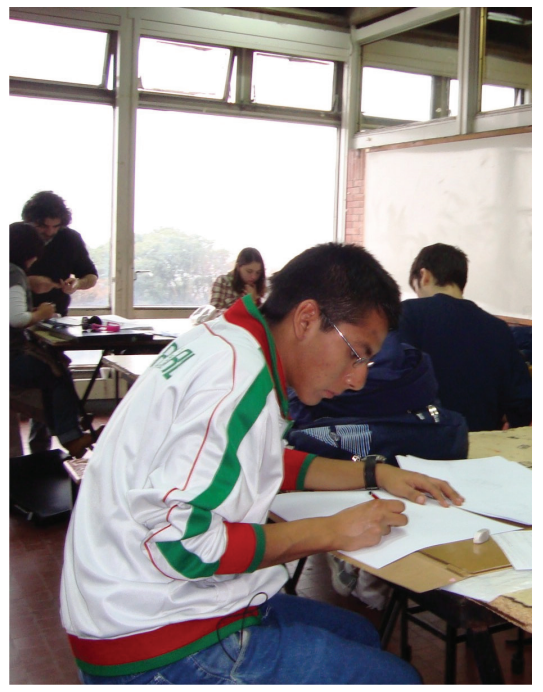
cada uno?

\section{MÉTODO}

\subsection{MUESTRA Y DISEÑO}

Se trabajó con una muestra de 300 sujetos, extraída de la población compuesta por alumnos que cursan el taller de Dibujo del Ciclo Básico Común en la Facultad de Arquitectura Diseño y Urbanismo de la Universidad de Buenos Aires. La muestra corresponde a dos cohortes de alumnos que cursaron el Taller de Dibujo en los períodos lectivos 2012 y 2013. Los sujetos tiene una edad media de 18,7 años (D.E: 2.39), un $67 \%$ son mujeres y un $33 \%$ son varones, proporción que refleja la composición de la población. 
Se usó un diseño transversal, no probabilístico, no experimental -no se manipularon variables-, de carácter intencional -porque se trabajó con los alumnos que cursaban la materia, sin establecer otro criterio de selección- y ecológico -ya que los datos se recogieron en el aula.

Para el análisis de los datos se usaron los siguientes estadísticos: prueba de significación de la diferencia de medias y análisis de cluster de K medias. Se usó el SPSS.20.

\subsection{INSTRUMENTOS}

La variable rendimiento en mano alzada corresponde a la evaluación de las láminas que componen el módulo de Sistemas de representación Sensible, con un puntaje de 0 a 10, que luego se convirtió en una variable dicotómica: de 0 a 5.50, rendimiento malo/regular; mayor a 5.50, rendimiento bueno/muy bueno.

Figura 1. Dibujos correspondientes al Módulo de Representación Sensible realizados por los alumnos durante el curso de Taller de Dibujo
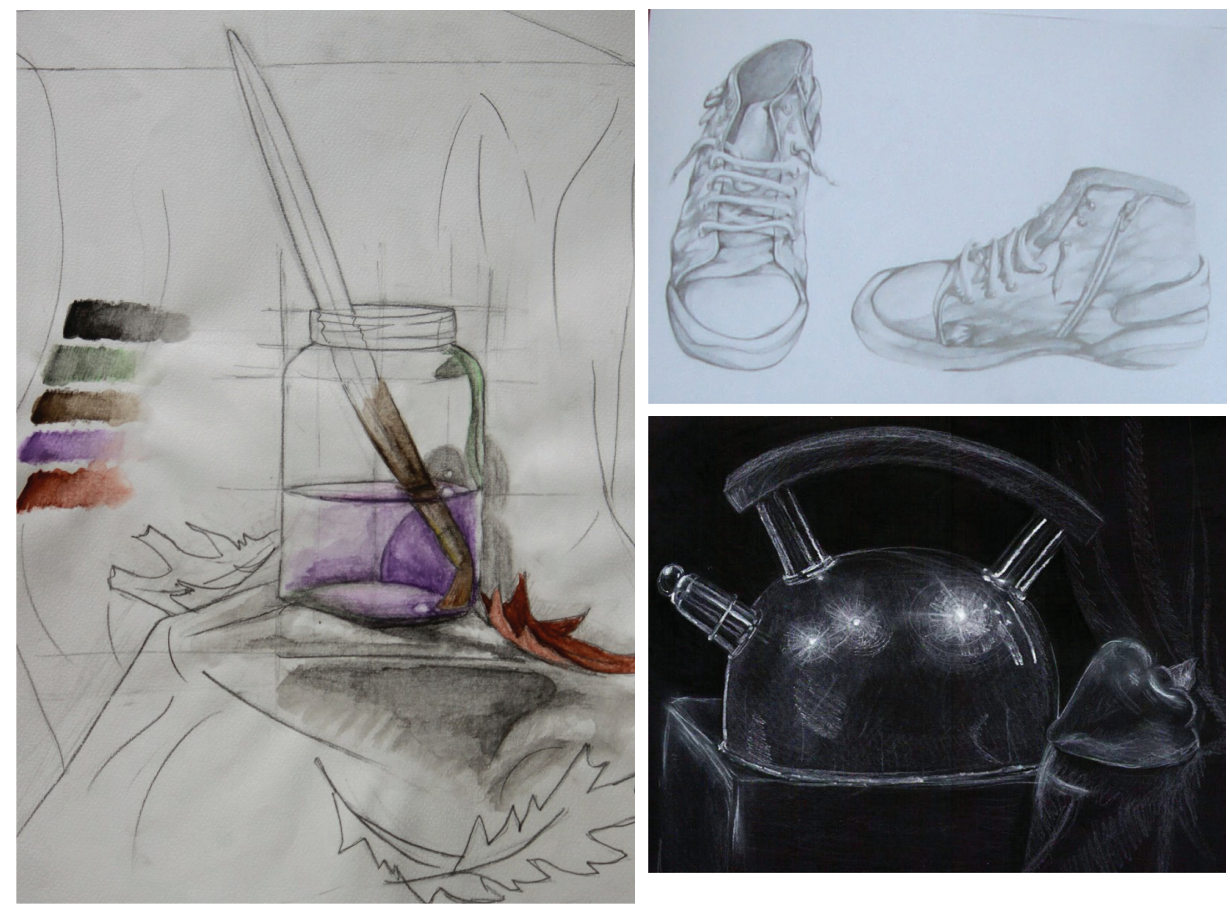

Los componentes de aprendizaje autorregulado se evaluaron con el MSLQ (Motivated Strategies for Learning Questionnaire, Pintrich y De Groot, 1990; García Duncan y McKeachie, 2005), un instrumento compuesto por 81 ítems distribuidos en 15 subescalas Likert que evalúan los componentes cognitivos, metacognitivos y afectivos que forman 
parte del comportamiento académico autorregulado: referidos al aspecto motivacional de expectativas y valor (metas intrínsecas y extrínsecas de la tarea académica, autoeficacia, creencias acerca de la importancia intrínseca de una tarea, y de su interés y utilidad para el sujeto; creencias de control de resultados y ansiedad); referidos a aspectos cognitivos y socio-cognitivos (uso de estrategias cognitivas de repetición, elaboración, organización y pensamiento crítico, planificación y monitoreo de la propia actividad, manejo del tiempo y ambiente de estudio, inversión de esfuerzo, inclinación al trabajo en grupo, y búsqueda de ayuda).

Para la asignación de puntaje, cada ítem del MSLQ se evalúa con una escala Likert (0 a 3), se suma el puntaje obtenido en cada ítem y el resultado se convierte a escala 10 . Al finalizar el primer semestre se entregó a cada alumno un cuestionario MSLQ, para que lo completaran fuera del horario de clases. Se recogieron 244 protocolos.

Los estilos de aprendizaje se obtuvieron a partir de un análisis de cluster de $\mathrm{K}$ medias, del que resultó la existencia de tres estilos:

Superficial: este grupo se caracteriza por el bajo empleo de estrategias cognitivas, muy poca capacidad de autorregulación, de manejo de tiempo y ambiente, y de motivación intrínseca.

Intermedio: es un grupo con un uso moderado de estrategias cognitivas y metacognitivas, con el nivel de ansiedad más alto.

Profundo: este grupo sobresale por sus metas intrínsecas y el valor que le da a la tarea académica, informan del uso de estrategias básicas y profundas, manejan su tiempo, ambiente y esfuerzo, y buscan ayuda.

Se administró, además, una encuesta en la que se pidió a los alumnos que evaluaran su habilidad para el dibujo a mano alzada, con cuatro categorías: mala, regular, buena, muy buena. Posteriormente, para los efectos de los análisis, se reagruparon estas categorías, creando una variable dicotómica, ya que el número de sujetos en las categorías extremas era bajo.

\section{RESULTADOS}

\subsection{CREENCIA EN LA PROPIA HABILIDAD}

Los datos analizados indican que el $45 \%$ de los sujetos se autoevaluaron como buenos o muy buenos en la habilidad del dibujo a mano alzada y sólo un $35 \%$ resultó tener este tipo de desempeño.

La verificación de la objetividad de la creencia requiere considerar en forma separada el grupo que se autoevaluó como bueno-muy bueno y el que se autoevaluó como malo-regular.

En el caso de los sujetos que se juzgan malos o regulares, se observó un porcentaje significativo de correspondencia entre la autoevaluación de la habilidad y el desempeño efectivo, tal como es evaluado por los docentes. Para la creencia positiva, en cambio, el grado de ajuste es menor $\mathrm{y}$, de hecho, resulta estadísticamente significativa la discrepancia $(\mathrm{Z}=4.62, \mathrm{p}<.0001)($ Ver Gráfico 1$)$. 
Gráfico 1. Relación entre creencia y desempeño en dibujo a mano alzada

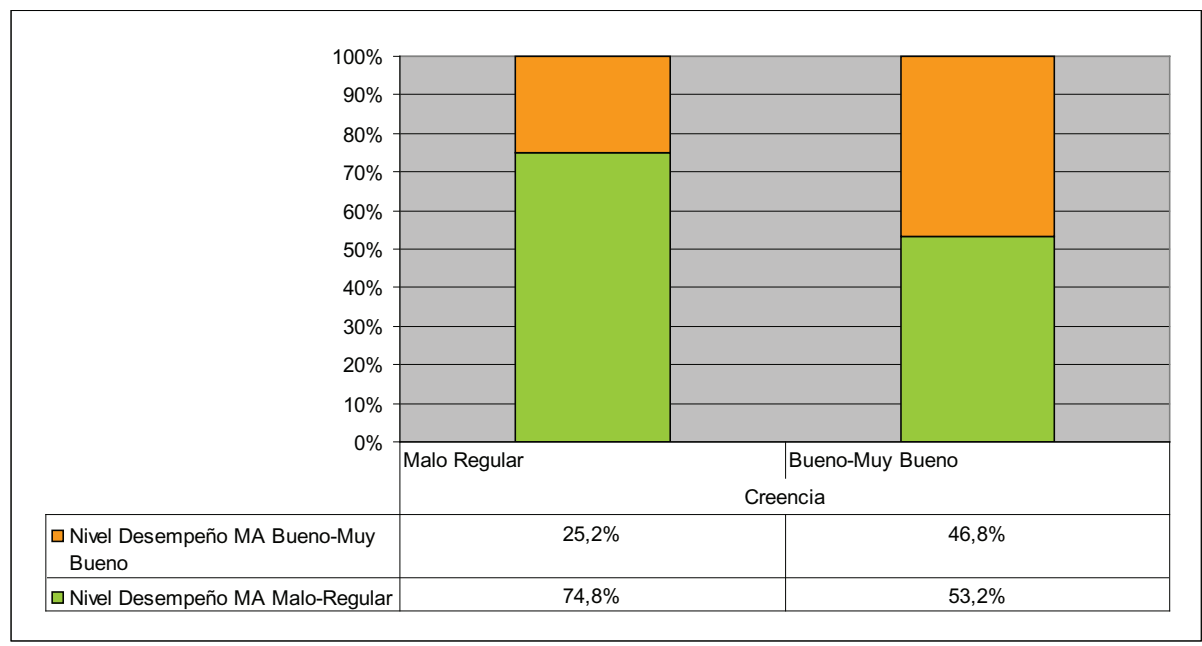

\subsection{ESTILOS DE APRENDIZAJE Y HABILIDAD EN DIBUJO A MANO ALZADA}

Se observa que dentro del grupo con rendimiento malo o regular en dibujo a mano alzada, hay un porcentaje alto de ajuste entre el juicio auto-evaluativo y el rendimiento efectivo, sin diferencias significativas por estilo de aprendizaje. En cambio, en el grupo con rendimiento bueno o muy bueno, el mayor porcentaje de ajuste se da en los alumnos con estilo de aprendizaje profundo, en tanto que la mayor discrepancia se verifica en los alumnos con estilo superficial, de los cuales, un $61.5 \%$ se sobreestima $(Z=-2.47, p<.05)$ (Ver Gráfico 2).

Por otra parte, los sujetos que se autoevalúan como malos o regulares $(\mathrm{M} / \mathrm{R})$ tienen una media significativamente más alta en ansiedad $[\mathrm{F}(1,299)=23,6, \mathrm{p}<.02]$.

Gráfico 2. Objetividad de la creencia y estilos de aprendizaje.

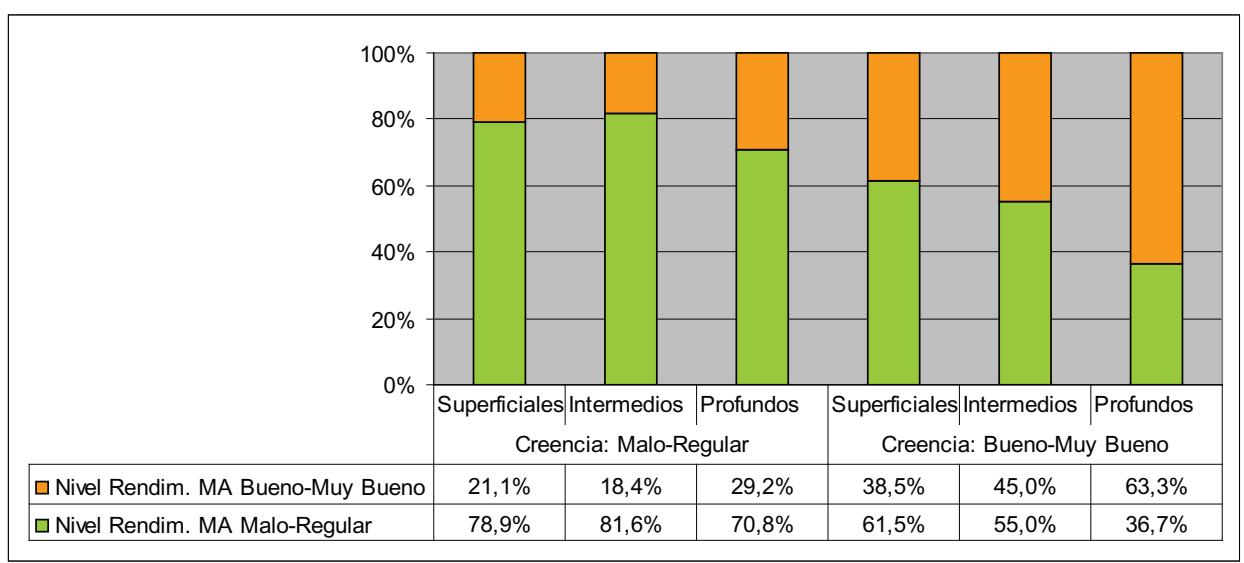




\section{CONCLUSIÓN}

La objetividad de la creencia en la propia habilidad se ha verificado parcialmente, para el caso del grupo de sujetos que se manifiestan negativamente al respecto. En cuanto al grupo que se evaluó erróneamente como bueno o muy bueno, este error parece ligarse con un estilo de aprendizaje superficial, lo que hace pensar que estos sujetos, menos inclinados a la reflexión, con niveles bajos de estrategias de procesamiento y de autorregulación, tienen también menos conciencia de sus límites. En este caso, la creencia en su autoeficacia, lejos de influir de modo positivo en su desempeño, es un obstáculo para su progreso académico.

La creencia objetiva positiva está relacionada con el estilo de aprendizaje profundo (metas intrínsecas, valor de la tarea, esfuerzo, recurso a la estrategia de pedir ayuda, elaboración, pensamiento crítico altos y ansiedad más baja), y a su vez esto se traduce en una mayor agentividad.

Se verifica así lo sostenido en otros estudios acerca de la influencia que la autoeficacia ejerce sobre el nivel de compromiso, pero cabe observar que si la creencia en la autoeficacia es errónea, dicha influencia se traduce en resultados negativos.

Por último, en el caso del grupo de los sujetos que se evalúan por debajo de su desempeño, esta creencia errónea no llega a afectar su agentividad, en la medida en que mantienen un nivel alto de valoración de la tarea académica e invierten alto nivel de esfuerzo, aunque manifiesten más ansiedad que el grupo cuya creencia es positiva. Esto mostraría que la creencia influye en este componente emocional del estilo de aprendizaje.

En definitiva, parecería que la agentividad depende más del estilo de aprendizaje que de la creencia en la autoeficacia, inferencia que permite hacer un aporte original al rol de la autoeficacia, dentro del contexto de los componentes del aprendizaje autorregulado.

En el aspecto pedagógico, el docente puede contribuir al desarrollo adaptativo de la creencia de autoeficacia, guiando al alumno en la toma de conciencia de sus propias posibilidades, promoviendo el empleo de estrategias propias del estilo profundo, haciendo que tome conciencia de la importancia del dibujo a mano alzada y su contribución al desarrollo del dominio de la espacialidad como requisito fundamental para el diseño.

Desde la perspectiva del fin de la educación, lo que llamamos estilo profundo se relaciona con rasgos psicológico-morales de la personalidad, ya que implica el ejercicio de la inteligencia y de la voluntad a partir de hábitos, como disposiciones virtuosas adquiridas, que permiten al alumno un autoconocimiento más objetivo y un compromiso con las tareas libremente asumidas. Esta observación permite ubicar el concepto de aprendizaje autorregulado en el contexto de la formación integral.

\section{REFERENCIAS BIBLIOGRÁFICAS}

Bandura, A. (1997). Self-efficacy: The exercise of control. New York: Freeman.

Boekaerts, M. \& Niemivirta, M. (2000). Self-regulated learning. Finding balance between learning goals and ego-protective goals. En M. Boekaerts; P. Pintrich \& M. Zeidner (Eds.), Handbook of self-regulation (417-450). San Diego. C. A.: Academic Press.

Boekaerts, M. \& Corno, L. (2005). Self-Regulation in the Classroom: A Perspective on Assessment and Intervention. Applied Psychology: An International Review, vol. 54, n.2, 199-231.

García Duncan, T. \& McKeachie, W. J. (2005). The making of the motivated strategies for learning questionnaire. Educational Psychologist, vol. 40, n.2, 117-128. 
Mancini, F.; Santoruvo, A.; Biondani, I. Battilana, E. e Carolo, M. (2013). La percezione di autoefficacia negli psicoterapeuti informazione: Un'indagine esplorativa. Cognitivismo clinico, vol. 10, n. 2, 173-184.

Pallasmaa, J. (2009). Tocando el mundo. Espacio vivencial, visión y hapticidad. Arquitecturas del Sur, vol. 36, 80-93. Recuperado de: http://issuu.com/arquitecturasdelsur/docs/as_36/83 [10/06/2014].

. (2005). The eyes of the skin. Architecture and the senses. Wiley-Academy: Chichester (WestSussex).

Pintrich, P. (2000). The role of goal orientation in self-regulated learning. En M. Boekaerts, P. Pintrich \& M. Zeidner (Eds) Handbook of self-regulation (451-502). San Diego. C.A: Academic Press

Pintrich, P. \& De Groot, E. V. (1990). Motivated and self-regulated learning components of classroom academic performance. Journal of Educational Psychology, vol. 82, n.1, 33-40.

Pintrich, P. \& Zusho, A. (2002). The development of academic self-regulation: The role of cognitive and motivational factors. En A. Wigfield \& J.S. Eccles (Eds.), Development of achievement motivation (249-284). San Diego, CA: Academic Press.

Schunk, D. H. (2005). Self-Regulated Learning: The Educational Legacy of Paul R. Pintrich. Educational Psychologist, vol. 40, n.2, 85-94.

Salanova, M. \& Llorens, S. (2011). "Yes, I can, I feel good, and I just do it". On gain cycles and spirals of efficacy beliefs, affect and engagement. Applied Psychology: An International Review, vol. 60, n. 2, 255-285.

Trachana, A. (2012). Manual o digital. Fundamentos antropológicos del dibujar y construir modelos arquitectónicos. Expresión Gráfica Arquitectónica (EGA), vol. 19, 289-300. Recuperado de: https://ojs.upv.es/index.php/EGA/article/view/1381/1404 [26/05/2014].

Zimmerman, B. J. (2000). Attaining self-regulation: A social cognitive perspective. En M. Boekaerts; P. Pintrich \& M. Zeidner (Eds.), Handbook of self-regulation (13-39). San Diego: Academic Press. 
